\title{
Molecular Docking of Anopheles gambiae and Aedes aegypti Glutathione S-Transferases Epsilon 2 (GSTE2) Against Usnic Acid: an Evidence of Glutathione Conjugation
}

\author{
Rafael Trindade Maia ${ }^{1 *}$ and Daniela Nadvorny ${ }^{2}$ \\ ${ }^{1}$ Departamento de Zoologia; Universidade Federal de Pernambuco. ${ }^{2}$ Departamento de Química Fundamental; \\ Universidade Federal de Pernambuco; Recife - PE - Brasil
}

\begin{abstract}
The aim of this study was to develop a theoretical model using Anopheles gambiae GSTE2 structure as template for Aedes aegypti GSTE2 by homology modeling Docking simulations were performed for both the enzymes against usnic acid in neutral and anionic forms. Ramachandran plot revealed that 93.9\% of the GSTE2 model residues were located on most favored regions. Model evaluation was made by the ANOLEA and GROMOS analysis. Docking results indicated that the enzymes were able to form glutathione-conjugate with usnic acid in both the forms (anionic and neutral).
\end{abstract}

Key words: Anopheles gambiae, Aedes aegypti, molecular modeling, insecticide resistance

\section{INTRODUCTION}

The Glutathione S-tranferases (GSTs) is a highly promiscuous enzyme super family that plays an essential role in cytoplasm detoxification of a large range of xenobiotic compounds in many organisms (Che-mendoza 2009). The GSTs displays multispecificity for substrate metabolism, involved in the catalysis of endogenous and xenobiotic compounds (Che-mendoza 2009). GSTs also function as non-enzymatic binding proteins (known as ligandins) participating in the intracellular transport (Listowsky et al. 1988) and signaling processes (Adler et al. 1999; Cho et al. 2001). This diversity of enzymatic and nonenzymatic functions is explained by the genetic capacity to encode different GST isoforms by the organisms (Che-mendoza 2009).

The main reaction catalyzed by the GSTs is the conjugation of the tripeptide glutathione (GSH) to a hydrophobic and cytotoxic compound, resulting in a new conjugate that is more soluble. In insects, there are six GSTs class described: Delta, Epsilon, Omega, Sigma, Theta and Zeta (Ding et al. 2003; $\mathrm{Tu}$ and Akgul 2005). The epsilon class is arthropod specific and is involved in insecticide metabolism and resistance. This GST class is represented at least by eight members in the mosquitoes: GSTE1, GSTE2, GSTE3, GSTE4, GSTE5, GSTE6, GSTE7 and GSTE8 (Ding et al. 2003).

In this protein superfamily, there is one specific enzyme, the GSTE2 that has been associated with the resistance to chemical insecticides on the mosquitoes Anopheles gambiae and Aedes aegypti, respectively the main malaria and dengue fever vectors (David et al. 2005; Lumjuan et al. 2007). The GSTE2 aminoacid sequence is available for both the species (Ding et al. 2003; Lumjuan et al. 2007), but only the An. gambiae GSTE2

*Author for correspondence: rafael.rafatrin@gmail.com 
(AgGSTE2) has its tridimensional structure solved by crystallography (Wang et al. 2008).

The usnic acid (UA) (Fig. 1) is a secondary metabolite found in several species of lichens (Ingo 2002). This compound has received attention due is its wide variety of pharmacological activities, such as antimicrobial, analgesic and anti-inflammatory (Vijayakumar et al. 2000; Luzina et al. 2010). The insecticidal activity of the AU has also been the subject of scientific studies. Cetin et al. (2008) investigated the insecticides

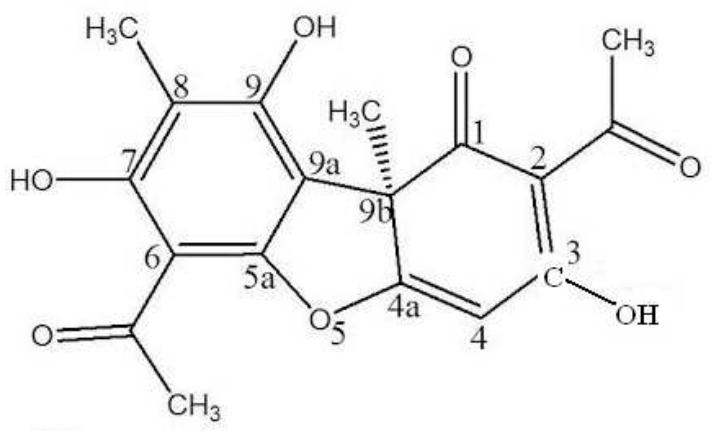

(a) effects of AU against Culex pipiens mosquito larvae and determined the insecticidal activity by bioassays.

In this study a homology model was built and evaluated for the A. aegypti glutathione Stransferase 2 (AaGSTE2). Docking simulations were performed where GSTE2 from Aedes aegypti and An. gambiae were used as receptors and anionic and neutral usnic acid forms were treated as ligand.

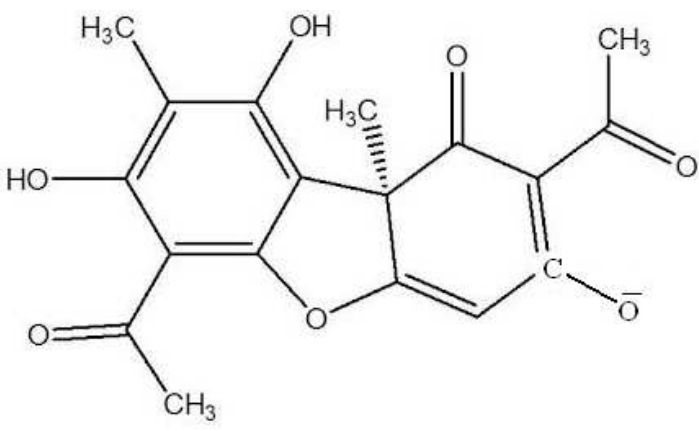

(b)

Figure 1 - Usnic acid in its (a) neutral and (b) anionic form.

\section{MATERIALS AND METHODS}

\section{Homology modelling of the AaGSTE2}

The AaGSTE2 and An. gambiae GSTE2 (AgGSTE2) amino acid sequences were obtained from Vectorbase data bank (AaGSTE2 ID number: AAEL007951-PA; AgGSTE2 ID number: AGAP009194-PA) and submitted to BLAST2p sequence (Altschul et al. 1997) for seeking the homology between them. The AgGSTE2 structure was obtained on PDB database (PDB ID: 2imi; Resolution: $1.4 \AA$ ) and was selected for template to build the AaGSTE2 model. The SWISSMODEL workspace (http://swissmodel.expasy.org/) was used to construct the structural model for the target sequence (Arnold et al. 2006). For structural optimization, the final model was submitted to an energy minimization on the Chiron web Server-http://troll.med.unc.edu/chiron/index.php (Ramachandran et al. 2011).

\section{Model evaluation and database submission} The AaGSTE2 was analyzed by PROCHECK (Laskowski et al. 1993) for the structure quality evaluation. Ramachandran diagram was requested to access the stereochemical quality. The ANOLEA mean force potential (Melo and Feytmans 1998) and the GROMOS force field (Scott et al. 1999) were used to evaluate the local quality of the structural model. After validation, the theoretical model was submitted to a public online repository, the PMDB - Protein Model Data base (its structure is available for public access under the ID code PM0079164).

\section{Preparation of receptor and ligand structures}

The initial coordinates of the AU were developed with the Gaussian 03 program (Frisch et al. 2004). The method B3LYP and $6-31+\mathrm{G}(\mathrm{d}, \mathrm{p})$ functions basis set was applied to calculate geometry of lower energy, which was submitted to the docking. The ligands were assigned with Gasteiger charge parameters (Gasteiger and Marsili 1980) and all polar hydrogen were removed. The AgGSTE2 and AaGSTE2 PDB files were prepared as receptors by adding hydrogen, assigning Kollman charges (Weiner et al. 1984) and converting to pdbqt files. The glutathione tripeptide was treated as co-factor. 


\section{Molecular docking simulations}

The docking experiment was performed on the Autodock 4.3.2 software (Morris et al. 2010). Docking simulations were run using Lamarckian Genetic algorithm (LGA). The grid points for Autogrid calculations were set to be $52 \times 52 \times 52$ $\AA$ with the active site residues at the center of the grid box. The docking parameters were set to a LGA calculation of 10,000 runs. The energy evaluations were set to $1,500,000$ and 27,000 generations. The Population size was set to 150 and the rate of gene mutation and the rate of gene crossover were set to 0.02 and 0.8 , respectively. The obtained conformations were then summarized, collected and extracted by using Autodock Tools. The first and the last conformation was analyzed from a 10-ranked set of each complex using the VMD-Visual Molecular Dynamics (Humpfrey et al. 1996).

\section{RESULTS AND DISCUSSION}

The protein Blast output revealed an excellent score (353 bits) and no gaps were found. The entire alignment is shown in Figure 2. The stereochemical quality of the predicted model was confirmed by the Ramachandran plot results (Fig. 3 ), whose $93.9 \%$ of residues were within the most favored regions. The local energy evaluation of the model by ANOLEA and GROMOS showed low energy values in most residues. Both ANOLEA and GROMOS energies showed negative values for the majority of protein residues. It indicated that they were located on favorable energy environment. These results suggested that the AaGSTE2 model displayed structural quality and reliability. It is the first structural model available for the AaGSTE2 enzyme, and the information about its structure could be very useful for further studies.

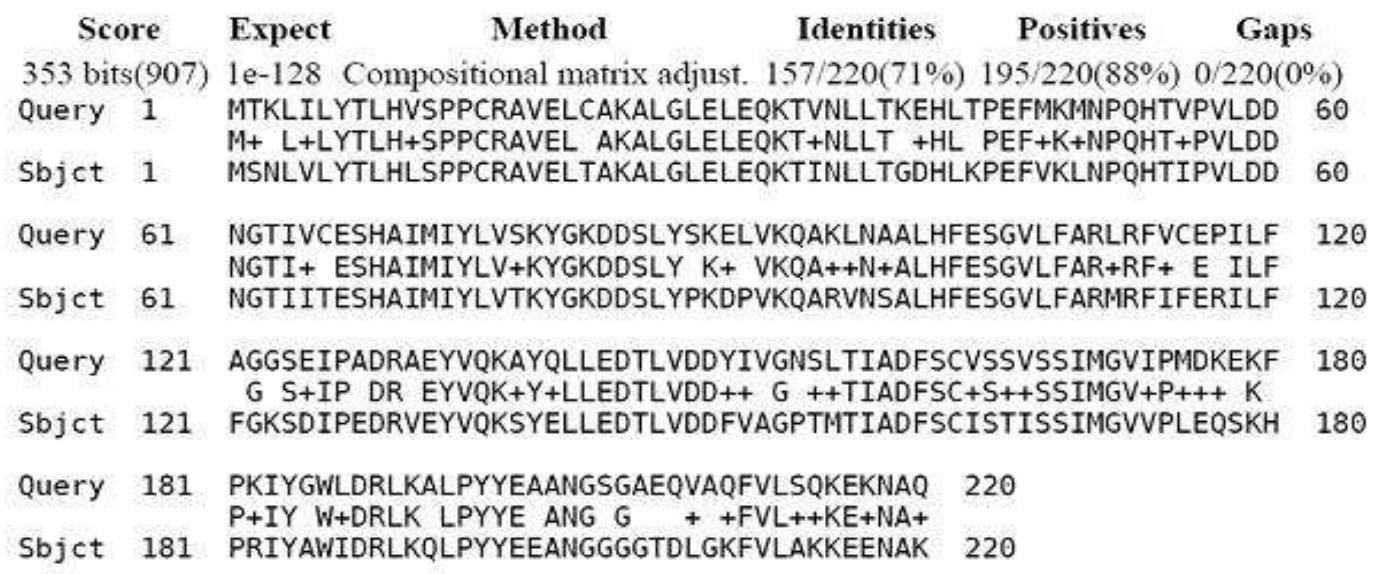

Figure 2 - BLAST 2p sequence output for AaGSTE2 (Query) and AgGSTE2 (Sbjct).

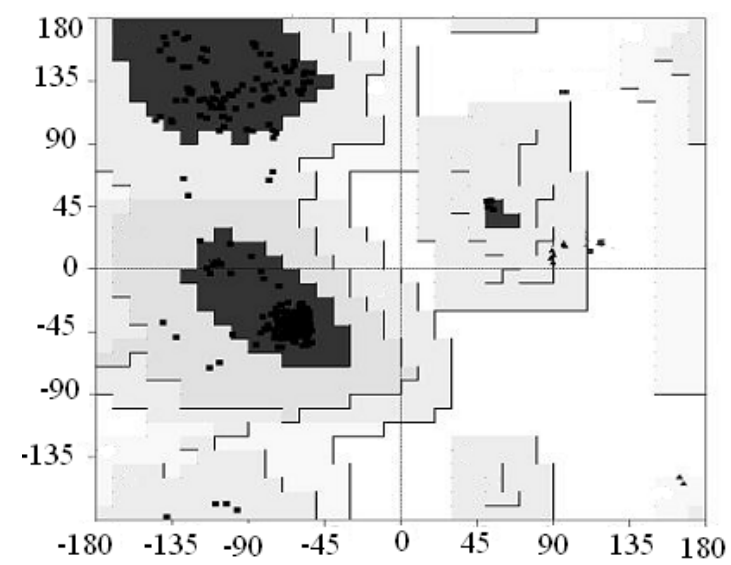

Figure 3 - Ramachandran plot for the AaGSTE2 predicted model.
Due the fact that X-rays structures were not available for AaGSTE2, a homology model was generated to perform the docking simulations. According to Laskowski et al (1993), predicted models are supposed to be reliable if over $90 \%$ of residues are situated in the core regions (Ramachandran plot). By this criterion, one could consider the stereochemical quality of the AaGSTE2 model (93.9\%) quite satisfactory.

The binding energies for all the complexes displayed negative values. The ANOVA test did not find significant difference ( $p>0.05$ ) among the docking energies from the neutral and anionic forms as well as it did not point statistical significance between the enzymes. The lowest 
energy value obtained was for the first conformation of AaGSTE2-UA deprotonated complex (Table 1). The highest energy was observed on the last conformation of the AaGSTE2-UA neutral complex (Table 1). Visual analysis showed the usnic acid involved in the Gsite pocket in all the conformations.

The distances between usnic acid and GSH are showed at Table 2. In all the complexes, the oxygen from UA was the atom that interacted with the glutathione, which interacted with both hydrogen and sulfur atoms (Fig. 4). The Pearson's correlation coefficient was negative and significant $(r=-0.786 ; \mathrm{p}<0.05)$, which showed that when AU oxygen-GSH hydrogen distances decreased, the AU-oxygen-GSH sulfur distances increased (Fig. 4).

Table 1 - Docking binding energies (Kcal/mol) of Anopheles gambie and Aedes aegypti GSTE2 against neutral and deprotonated usnic acid forms.

\begin{tabular}{lcc}
\hline Enzime & $\begin{array}{c}\text { Usnic acid } \\
\text { (neutral) }\end{array}$ & $\begin{array}{c}\text { Usnic acid } \\
\text { (anion) }\end{array}$ \\
\hline AaGSTE2-conformation 1 & -7.11 & -7.63 \\
AgGSTE2-conformation 1 & -5.78 & -6.37 \\
AaGSTE2-conformation 10 & -4.95 & -7.37 \\
AgGSTE2-conformation 10 & -6.95 & -5.51 \\
\hline
\end{tabular}

Table 2 - Distances between UA oxygen and GSH hydrogen and sulfur atoms. The parenthesis shows distances in the last conformation, while other values refer to the first conformation of each complex in the docking energy ranking.

\begin{tabular}{lcc}
\hline Binding & $\begin{array}{c}\text { Distance } \AA \\
\text { O-H-GSH }\end{array}$ & $\begin{array}{c}\text { Distance } \AA \\
\text { O-S-GSH }\end{array}$ \\
\hline AgGSTE2-AU & 2.19 & 3.40 \\
neutral & $(1.85)$ & $(3.69)$ \\
AgGSTE2-AU & 2.19 & 3.44 \\
deprotonated & $(1.81)$ & $(4.45)$ \\
AaGSTE2-AU & 1.89 & 3.86 \\
neutral & $(1.85)$ & $(3.94)$ \\
AaGSTE2-AU & 1.97 & 3.73 \\
deprotonated & $(2.09)$ & $(3.77)$ \\
\hline
\end{tabular}

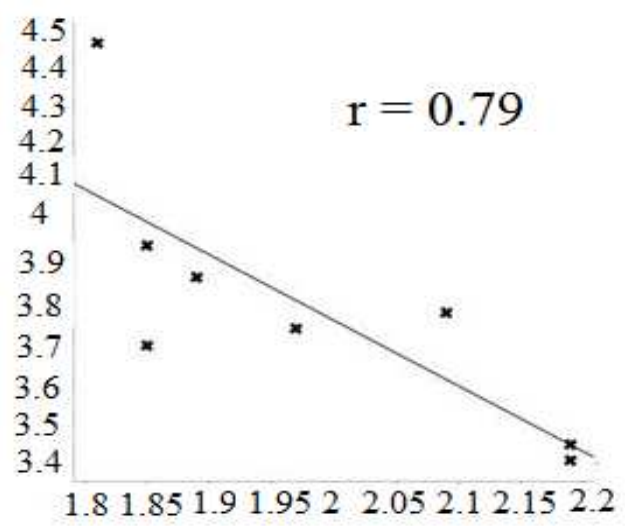

Figure 4 - Plot of AU oxygen-GSH hydrogen distances (X-axis) versus AU-oxygen-GSH sulfur distances (Y-axis). Pearson's coefficient (r value) are shown.

Results suggested that the UA-GSH conjugate was formed by An. gambiae and A. aegypti GSTE2 activity (Fig. 5). These enzymes should also be able to metabolize the both usnic acid forms: the neutral and anionic (Fig. 6 and 7). These results were the first evidence of usnic acid conjugation by an insect glutathione S-transferase and the first in-silico docking study with this compound.

Docking studies have already been done for the AgGSTE2 against DDT and DDE (Setzer 2011). Others detoxification enzyme families had also been docked against the insecticide in Anopheles mosquito (Chiu et al. 2008). The information about how detox enzymes binds to insecticidal compounds could be extremely useful for specific inhibitors development. The present results showed that both usnic acid forms bound to the proteins, which meant there was a real possibility that AaGSTE2 and AgGSTE2 could metabolize this natural insecticide. These enzymes are promising targets for design new technologies tools as biosensors for the direct monitoring of environmental pollutants, such as insecticides (Chronopoulou et al. 2009). Despite the proven larvicidal activity of usnic acid, this in silico study showed that AaGSTE2 and AgGSTE2 enzymes could be involved in usnic acid detoxification. 


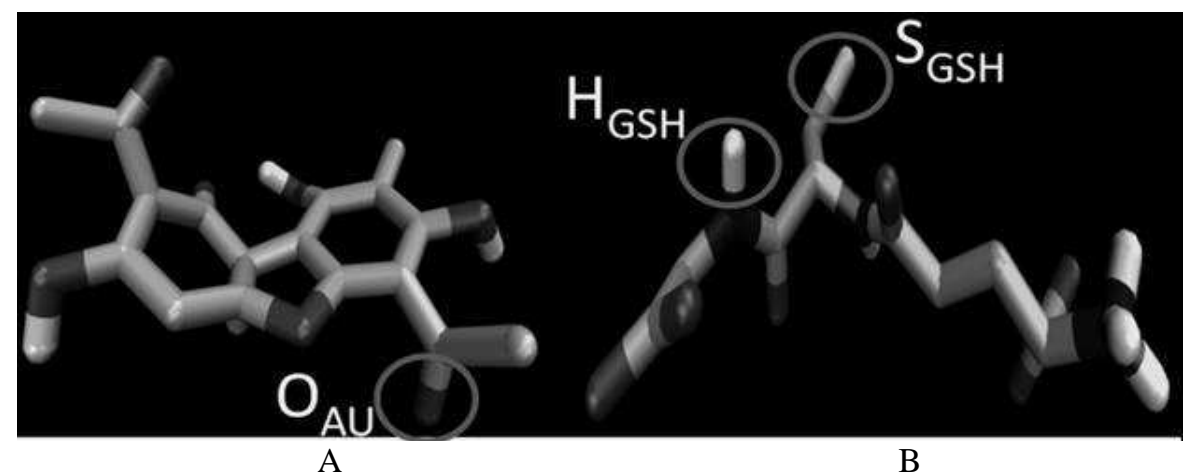

Figure 5 - The binding atoms for the ligands. $\mathrm{O}_{\mathrm{AU}}=$ usnic acid oxygen; $\mathrm{H}_{\mathrm{GSH}}=$ glutathione hydrogen; $\mathrm{S}_{\mathrm{GSH}}=$ glutathione sulfur.

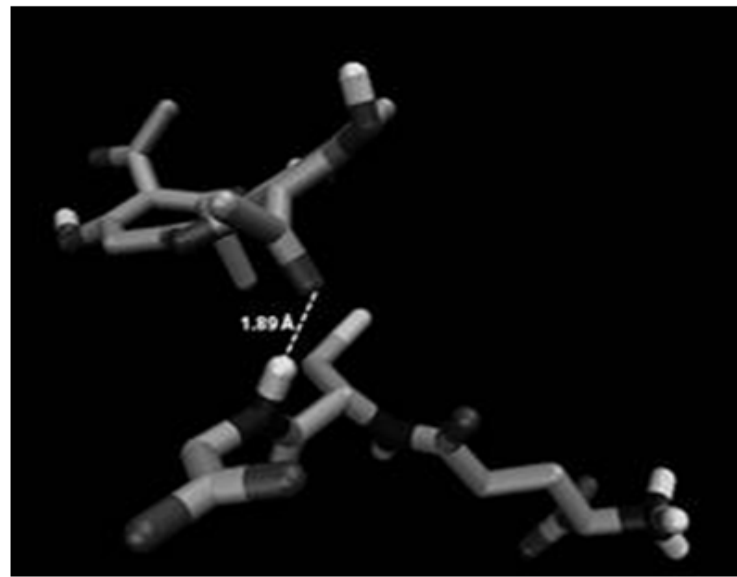

A

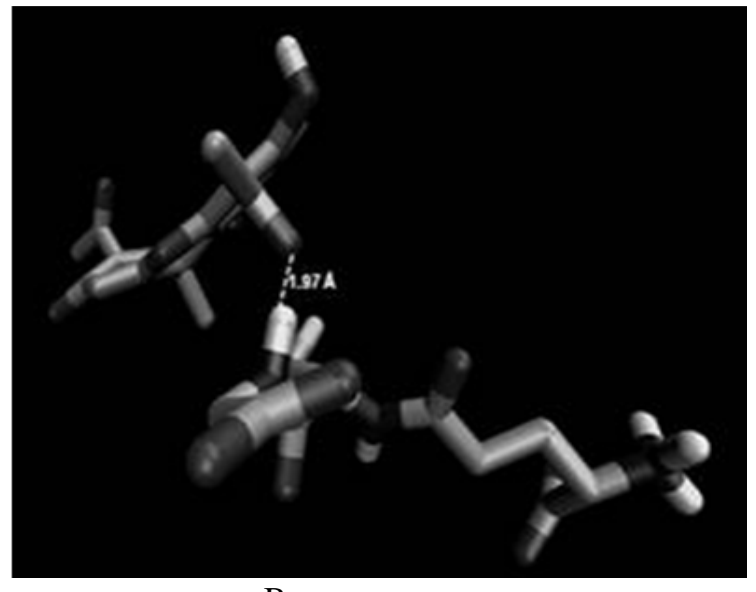

B

Figure 6 - Best energy ranked conformations for AaGSTE2-AU netral (A) and AaGSTE2-AU anionic (B) complexes.
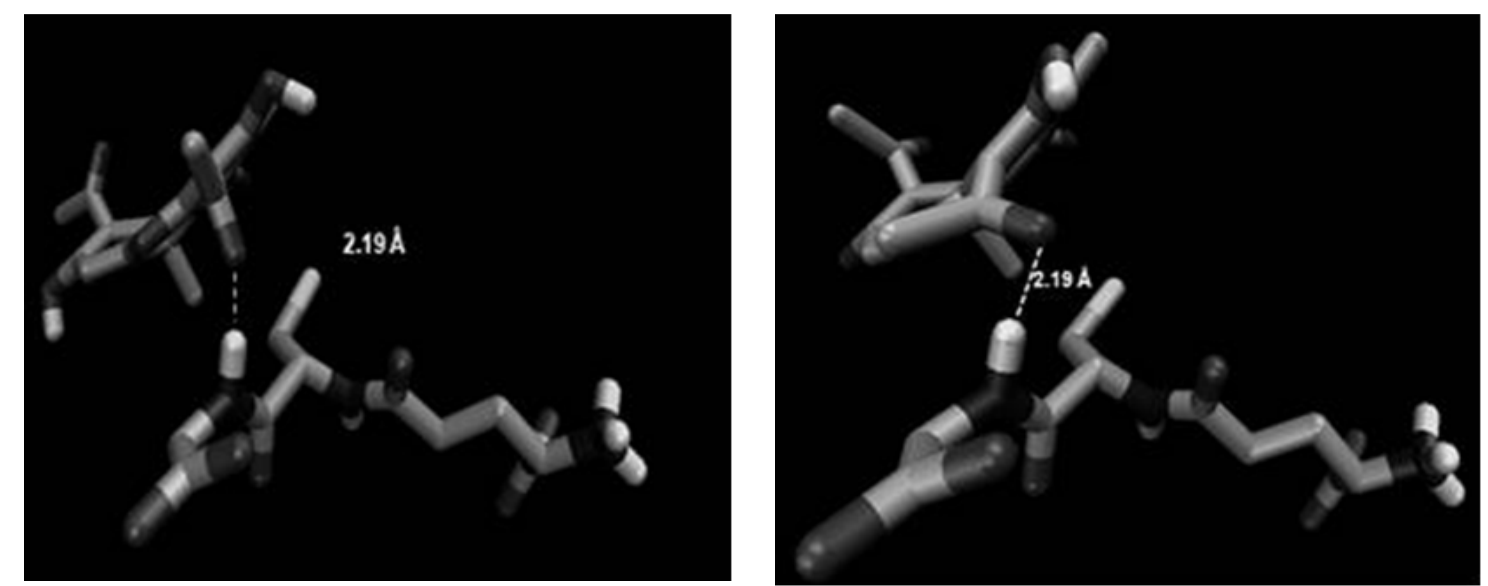

Figure 7 - Best energy ranked conformations for AaGSTE2-AU netral (A) and AaGSTE2-AU anionic (B) complexes.

\section{CONCLUSIONS}

The modeling results led to conclude that the predicted model was representative for AaGSTE2, and that it was closely related to the homologous
AgGSTE2. This homology relationship was supported by their sequence identity and similarity, structural features and affinity to the substrates. The docking results showed that both 
the enzymes (AaGSTE2 and AgGSTE2) displayed a relevant role on enantiomeric usnic acid forms metabolism. Development of a resistance way to usnic acid could results the use of this compound as an insecticide as a safe and efficient for mosquito control in future.

\section{ACKNOWLEDGMENT}

We thank to Biomat group for providing computational resources for this study.

\section{REFERENCES}

Altschul SF, Madden TL, Schaffer AA, Zhang JH, Zhang Z, Miller W, et al. Gapped BLAST and PSIBLAST: a new generation of protein database search programs. Nucleic Acids Res. 1997; 25: 3389.

Arnold K, Bordoli, L, Kopp, J, Schwede T. The SWISS-MODEL workspace: a web-based environment for protein structure homology modelling. Bioinformatics. 2006; 22: 195.

Cetin H, Tufan-Cetin O, Turk AO, Tay T, Candan M, Yanikoglu A, et al. Insecticidal activity of major lichen compounds, (-)- and (+)- usnic acid, against the larvae of house mosquito, Culex pipiens L. Parasitol Res. 2008; 102: 1277-1279.

Che-mendoza A, Penilla RP, Rodríguez DA. Insecticide resistance and glutathione S-transferases in mosquitoes: A review. Afr J Biotechnol. 2009; 8: 1386-1397.

Chiu TL, Wen Z, Rupasinghe SG, Schuler MA. Comparative molecular modeling of Anopheles gambiae CYP6Z1, a mosquito P450 capable of metabolizing DDT. Proc Natl Acad Sci. 2008: 105, 8855-8860.

Chronopoulou EG, Labrou, NE. Glutathione Transferases: Emerging Multidisciplinary Tools in Red and Green Biotechnology. Science. 2009; 3: 211223.

David J, Strode C, Vontas J, Nikou D, Vaughan A, Pignatelli PM, et al. The Anopheles gambiae detoxification chip: A highly specific microarray to study metabolic-based insecticide resistance in malaria vectors. $P$ Natl Acad Sci. 2005; 102:4080-4084.

Ding Y, Ortelli F, Rossiter LC, Hemingway J, Ranson H. The Anopheles gambiae glutathione transferase supergene family: annotation, phylogeny and expression profiles. BMC Genomics. 2003; 4: 35.

Frisch MJ, Trucks GW, Schlegel HB, Scuseria GE, Robb MA, Cheeseman JR, et al. Gaussian 03, 2004, Gaussian Inc., Wallinford CT.

Gasteiger J, Masili, M. Tetrahedron.1980, 36: 32193228.
Humphrey W, Dalke A, Schulten K.VMD: visual molecular dynamics. J Mol Graphics. 1996; 14: 2728.

Ingo K. Usnic acid. Phytochemistry. 2002; 61: 729-736.

Laskowski RA, MacArthur MW, Moss DS, Thornton, JM. PROCHECK: a program to check the stereochemical quality of protein structures. $J$ Appl Crystallogr. 1993; 26: 283-291.

Listowsky I, Abramovitz M, Homma H, Niitu Y. Intracelullar binding and transport of hormone and xenobiotics by glutathione S-transferases. Drug Metab Rev. 1988; 19: 305-318.

Lumjuan N, Stevenson BJ, Prapanthadara LA, Somboon P, Brophy PM, Loftus BJ et al. The Aedes aegypti glutathione transferase family. Insect Biochem Molec. 2007; 37: 1026-1035.

Luzina OA, Polovinka MP, Salakhutdinov NF, Tolstikov GA. Chemical modification of usnic acid: III. Reaction of (+)-usnic acid with substituted phenylhydrazines. Russ J Org Chem. 2010; 45: 1783451789.

Melo F, Feytmans E. Assessing protein structures with a non-local atomic interaction energy. $\mathrm{J} \mathrm{Mol} \mathrm{Biol.}$ 1998; 277: 1141-1152.

Morris GM, Huey R, Lindstrom W, Sanner MF, Belew RK, Goodsell DS, et al. AutoDock4 and AutoDockTools4: Automated docking with selective receptor flexibility. J Comput Chem. 30 (6): 27852791.

Ramachandran S, Kota P, Ding F, Dokholyan NV. Chiron. Proteins: Struct Funct Bioinf. 2011; 79: 261270.

Scott WRP, Hunenberger PH, Tironi IG, Mark AE, Billeter SR, Fennen J, et al. Biomolecular Simulation Package. J Phys Chem A. 1999; 103: 3596.

Setzer, WN. The molecular mechanism for DDT detoxification in Anopheles gambiae: a molecular docking study. J Biophys Chem. 2011; 2: 134-135.

Tu CPD, Akgul B. Drosophila glutathione Stransferases. Method Enzymol. 2005; 401: 204-226.

Vijayakumar CS, Viswanathan S, Reddy MK, Parvathavarthini S, Kundu B, Sukumar E. Antiinflammatory activity of (+) - usnic acid. Fitoterapia. 2000; 71: 564-566.

Weiner SJ, Kollman PA, Case DA, Singh UC, Ghio C, Alagona $G$, et al. A new force field for molecular mechanical simulation of nucleic acids and proteins. J Am Chem Soc. 1984; 106: 765-784.

Received: August 01, 2013; Accepted: April 22, 2014. 